\title{
Expression of miR-200a and chemotherapeutic treatment efficacy of glioma
}

\author{
$\mathrm{CHAO}_{\mathrm{WANG}}{ }^{1}, \mathrm{LE} \mathrm{KANG}^{2}, \mathrm{XIPENG} \mathrm{WANG}^{3}{ }^{3}{\text { YANPING } \text { LIU }^{3} \text { and XIA ZHAO }}^{4}$ \\ ${ }^{1}$ Emergency Intensive Care Unit; ${ }^{2}$ Operating Room; Departments of ${ }^{3}$ General Surgery and ${ }^{4}$ Nursing, \\ The Sixth People's Hospital of Jinan, Jinan, Shandong 250200, P.R. China
}

Received December 8, 2017; Accepted February 2, 2018

DOI: $10.3892 / \mathrm{ol} .2018 .8063$

\begin{abstract}
The correlation between miR-200a expression and chemotherapeutic treatment efficacy of glioma was investigated. There were 45 patients with glioma in observation group whose cancer tissues, paracancerous tissues and serum samples were harvested. Additionally, there were 23 healthy subjects in the control group whose serum samples were also collected. The expression levels of miR-200a in cancer tissues, paracancerous tissues and serum samples were measured by real-time fluorescence-based quantitative polymerase chain reaction (qRT-PCR). The t-test and one-way ANOVA were used to compare the serum levels of miR-200a in patients with different clinical features involving age, sex, tumor location, pathological grade and tumor size. All patients in the observation group received temozolomide-based chemotherapy. The serum levels of miR-200a before chemotherapy were compared between patients who were responsive to chemotherapy [complete response (CR) and partial response (PR)] and patients who were not responsive to chemotherapy [stable disease (SD) and progressive disease (PD)]. The expression level of miR-200a in cancer tissue was significantly lower than that in paracancerous tissue $(\mathrm{P}<0.05)$. The serum level of miR-200a in patients in the observation group was lower than that in healthy subjects in the control group $(\mathrm{P}<0.05)$. No correlations were found between miR-200a expression and patient age, sex and tumor location $(\mathrm{P}>0.05)$, but miR-200a expression was found to correlate with pathological grade and tumor size $(\mathrm{P}<0.05)$. The expression levels of miR-200a in serum and cancer tissue in chemotherapy-non-responsive patients (SD and $\mathrm{PD}$ ) were lower than those in chemotherapyresponsive patients (CR and $\mathrm{PR}, \mathrm{P}<0.05)$. The serum levels of miR-200a in chemotherapy-responsive patients were lower than those in healthy subjects in the control group $(\mathrm{P}<0.05)$. Downregulation of miR-200a was associated with onset and
\end{abstract}

Correspondence to: Dr Le Kang, Operating Room, The Sixth People's Hospital of Jinan, 1920 Huiquan Road, Jinan, Shandong 250200, P.R. China

E-mail:kx50b5@163.com

Key words: glioma, Ki-67, p53, miR-200a, temozolomide progression of glioma, and changes of miR-200a expression levels in patients were correlated with chemotherapeutic treatment efficacy.

\section{Introduction}

Glioma is a common intracranial malignant tumor, accounting for more than $50 \%$ of intracranial tumors (1). Gliomas are classified into four grades (I, II, III and IV) based on the 2016 World Health Organization (WHO) classification system. Grade I and grade II tumors are termed low-grade gliomas, and grade III and grade IV tumors are termed high-grade gliomas (2). Glioma has high disability and mortality rates associated with high incidence of metastasis and relapse. It is one of the major causes of disability and death in patients. Currently, glioma is treated mainly by surgery. However, the cancerous tissue is hard to be removed completely by surgery due to the infiltrative growth nature of glioma. A relapse often occurs after surgery (3). In addition, a number of challenges stand in the way of successful treatment for glioma, including sensitive tumor location, the blood-brain barrier that restrains anticancer drugs from entering into brain and tumor tissue, and drug resistance of cancer cells in glioma (4). There is evidence suggesting miRNA might render drug-resistant cancer cells in glioma sensitive to chemotherapy through modulation of target gene expression, thus enhancing chemotherapeutic efficacy (5). miR-200a is a member of the miR-200 family. Its loss is closely associated with onset of multiple cancers, and upregulated miR200 a expression is beneficial in inhibition of tumor growth (6). In the present study, the expression levels of miR-200a in cancer tissue, paracancerous tissue and serum were determined using real-time fluorescence-based quantitative polymerase chain reaction (qRT-PCR). Based on the acquired data, the correlation between miR-200a expression and chemotherapeutic treatment efficacy of glioma was explored.

\section{Materials and methods}

Subjects. Forty-five patients with glioma treated in the Sixth People's Hospital of Jinan from March 2015 to June 2017 were enrolled in this study as observation group. There were 19 males and 26 females, aged 26-68 years, with an average age of $52.3 \pm 9.6$ years. All patients with glioma underwent surgical resection. The cancer tissue and paracancerous tissue were 
harvested and stored in liquid nitrogen immediately. Patient's serum samples were also collected before chemotherapy treatment. In the meantime, serum samples were collected from 23 healthy subjects of similar age as control group who took routine physical examination in the Sixth People's Hospital of Jinan during the same time period.

Inclusion and exclusion criteria of patients. Inclusion criteria: Patients had a high-grade glioma identified by histopathological assay; expected survival was $>1$ year; the Karnofsky Performance Score (KPS) was $>60$ points. Exclusion criteria: Patients recently received immunotherapy; patients had a history of another kind of malignant cancer; patients had serious heart, liver, spleen, lung, or kidney diseases. The present study was approved by the Ethics Committee of the Sixth People's Hospital of Jinan (Jinan, China). Signed written informed consents were obtained from the patients and/or guardians.

Chemotherapy and efficacy evaluation. Forty-five patients with glioma in the observation group received temozolomidebased chemotherapy (SFDA Approval no. H20143117; Jiangsu Sheng Di Pharmaceutical Co., Ltd.). Temozolomide was administered in normal saline via IV injection at a dose of $75 \mathrm{mg} / \mathrm{m}^{2}$ once a day. Four weeks constitute a course of treatment. The chemotherapeutic efficacy was evaluated after 5 months of treatment according to the WHO response evaluation criteria. Complete response (CR) was defined as complete disappearance of the tumor with no new lesions. The response should last for at least 4 weeks. Partial response (PR) was defined as at least $50 \%$ reduction in the tumor size with no new lesions. The response should last for at least 4 weeks. Stable disease (SD) represents $<50 \%$ reduction to $<25 \%$ increase in the tumor size. Progressive disease (PD) represents at least $25 \%$ increase in the tumor size or appearance of new lesions.

Total RNA extraction and cDNA synthesis. Total RNA was extracted from tissues and serum using the TRIzol kit (Takara Bio, Dalian, China) and the mirVana PARIS kit (Shanghai Ponsure Biotechnology, Inc., Shanghai, China) in accordance with the manufacturer's instructions. RNA purity and concentration were determined by spectrophotometry at 260 and $280 \mathrm{~nm}$. cDNA synthesis was performed by reverse transcription of $1 \mu \mathrm{g}$ RNA using the PrimeScript ${ }^{\mathrm{TM}}$ RT Master Mix kit (Takara Bio) in accordance with the manufacturer's instruction.

Determination of miR-200a expression levels by qRT-PCR. The qRT-PCR assay was performed using an ABI 7100 realtime fluorescence-based quantitative PCR instrument. The PCR reaction system contained $1.0 \mu \mathrm{l}$ of cDNA, $0.8 \mu \mathrm{l}$ of primer, $10 \mu \mathrm{l}$ of $2 \mathrm{X}$ miRNA qPCR Mix, and $8.2 \mu \mathrm{l}$ of $\mathrm{dd}_{2} \mathrm{O}$. The PCR reaction was run as follows: pre-denaturation at $95^{\circ} \mathrm{C}$ for $10 \mathrm{~min}$, followed by 45 cycles of $95^{\circ} \mathrm{C}$ for $10 \mathrm{sec}, 60^{\circ} \mathrm{C}$ for $20 \mathrm{sec}$ and $72^{\circ} \mathrm{C}$ for $12 \mathrm{sec}$. Using U6 as the reference gene, the expression level of miR-200a was calculated by the method of relative quantification $2^{-\Delta \Delta \mathrm{Cq}}$. Specific primer sequences are as follows: for miR-200a the forward sequence is TAA CAC TGT CTG GTA ACG ATG T and the reverse sequence is ATC GTT ACC AGA CAG TGT TAT T; for U6 the forward sequence is
Table I. Comparison of the general characteristics between the observation group and the control group.

\begin{tabular}{lcccc}
\hline Items & $\begin{array}{c}\text { Observation } \\
\text { group }\end{array}$ & $\begin{array}{c}\text { Control } \\
\text { group }\end{array}$ & P-value & t-value \\
\hline Sex & & & & \\
$\quad$ Male & 19 & 10 & 0.190 & 1.674 \\
Female & 26 & 13 & & \\
Age (years) & & & & \\
$\leq 50$ & 29 & 14 & 0.127 & 1.765 \\
$>50$ & 16 & 9 & & \\
\hline
\end{tabular}
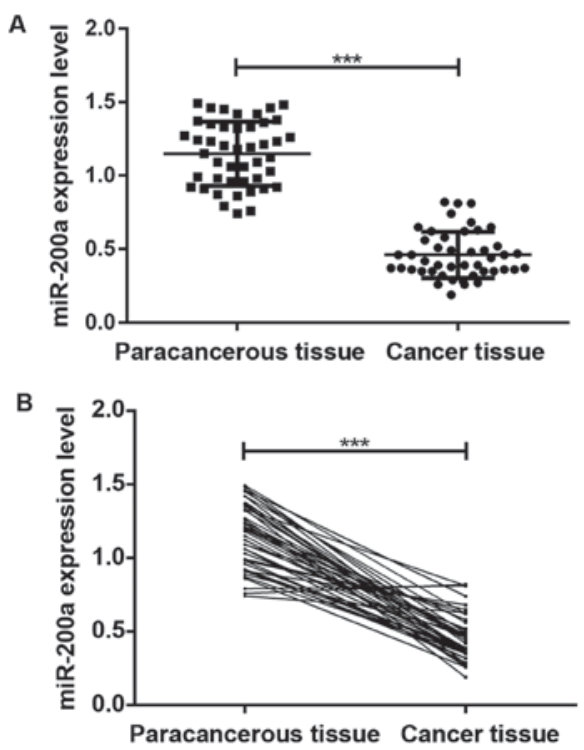

Figure 1. Determination of miR-200a expression levels in cancer tissue and paracancerous tissue by qRT-PCR. The expression level of miR-200a in cancer tissue of patients with glioma was significantly lower than that in paracancerous tissue. ${ }^{* * *} \mathrm{P}<0.001$

CTC GCT TCG GCA GCA CAT TTT and the reverse sequence is AAC GCT TCA CGA ATT TGC GT. Primers were synthesized by Shanghai Sangon Biotech Co., Ltd. (Shanghai, China).

Statistical analysis. Data were statistically analyzed by SPSS 17.0 software (SPSS, Inc., Chicago, IL, USA). The t-test was used for comparison of differences between two groups. One-way ANOVA was used for comparison of differences between the above groups. Differences were considered statistically significant at $\mathrm{P}<0.05$.

\section{Results}

General characteristics. The t-test was used to analyze the differences of age and sex between the observation group and the control group. The results in Table I show that the differences of age and sex between the two groups were not statistically significant $(\mathrm{P}>0.05)$.

miR-200a expression levels in cancer tissue and paracancerous tissue. The results of qRT-PCR assay in Fig. 1 show 
Table II. Correlation between miR-200a expression level and clinical features of glioma patients.

\begin{tabular}{|c|c|c|c|c|c|c|c|}
\hline \multirow[b]{2}{*}{ Clinical feature } & \multirow[b]{2}{*}{$\mathrm{n}$} & \multicolumn{3}{|c|}{ Cancer tissue } & \multicolumn{3}{|c|}{ Serum } \\
\hline & & $\begin{array}{l}\text { Relative expression } \\
\text { level of miR-200a }\end{array}$ & P-value & t-value & $\begin{array}{l}\text { Relative expression } \\
\text { level of miR-200a }\end{array}$ & P-value & t-value \\
\hline \multicolumn{8}{|l|}{ Age (years) } \\
\hline$\leq 50$ & 29 & $0.41 \pm 0.08$ & \multirow[t]{2}{*}{$>0.05$} & \multirow[t]{2}{*}{0.1218} & $0.35 \pm 0.04$ & \multirow[t]{2}{*}{$>0.05$} & \multirow[t]{2}{*}{0.9015} \\
\hline$>50$ & 16 & $0.45 \pm 0.03$ & & & $0.36 \pm 0.07$ & & \\
\hline \multicolumn{8}{|l|}{ Sex } \\
\hline Male & 19 & $0.40 \pm 0.11$ & \multirow[t]{2}{*}{$>0.05$} & \multirow[t]{2}{*}{0.9145} & $0.32 \pm 0.06$ & \multirow[t]{2}{*}{$>0.05$} & \multirow[t]{2}{*}{0.3419} \\
\hline Female & 26 & $0.47 \pm 0.06$ & & & $0.36 \pm 0.02$ & & \\
\hline \multicolumn{8}{|c|}{ Pathological grade } \\
\hline III & 12 & $0.53 \pm 0.05$ & \multirow[t]{2}{*}{$<0.05$} & \multirow[t]{2}{*}{0.0126} & $0.39 \pm 0.05$ & \multirow[t]{2}{*}{$<0.05$} & \multirow[t]{2}{*}{0.0063} \\
\hline IV & 33 & $0.33 \pm 0.04$ & & & $0.28 \pm 0.03$ & & \\
\hline \multicolumn{8}{|c|}{ Tumor diameter $(\mathrm{cm})$} \\
\hline$\leq 5$ & 30 & $0.51 \pm 0.07$ & \multirow[t]{2}{*}{$<0.05$} & \multirow[t]{2}{*}{0.0083} & $0.34 \pm 0.04$ & \multirow[t]{2}{*}{$<0.05$} & \multirow[t]{2}{*}{0.0168} \\
\hline$>5$ & 15 & $0.42 \pm 0.09$ & & & $0.26 \pm 0.04$ & & \\
\hline \multicolumn{8}{|l|}{ Tumor location } \\
\hline Frontal lobe & 16 & $0.41 \pm 0.06$ & \multirow[t]{3}{*}{$>0.05$} & \multirow[t]{3}{*}{1.2462} & $0.31 \pm 0.06$ & \multirow[t]{3}{*}{$>0.05$} & \multirow[t]{3}{*}{0.8761} \\
\hline Temporal lobe & 20 & $0.39 \pm 0.04$ & & & $0.29 \pm 0.03$ & & \\
\hline Occipital lobe & 9 & $0.43 \pm 0.04$ & & & $0.33 \pm 0.01$ & & \\
\hline
\end{tabular}

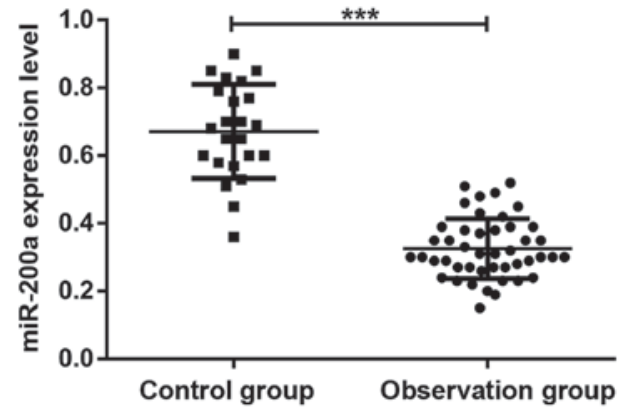

Figure 2. Serum miR-200a expression levels in patients with glioma before chemotherapy and healthy subjects in the control group. The serum level of miR-200a in patients with glioma before chemotherapy was significantly lower than that in healthy subjects. ${ }^{* * *} \mathrm{P}<0.001$.

that the expression level of miR-200a in cancer tissue of patients with glioma was significantly lower than that in paracancerous tissue $(\mathrm{P}<0.05)$, and the difference was statistically significant.

Serum miR-200a expression levels in patients with glioma before chemotherapy and healthy subjects in the control group. As shown in Fig. 2, the serum level of miR-200a in patients with glioma before chemotherapy was significantly lower than that in healthy subjects $(\mathrm{P}<0.05)$ and the difference was statistically significant.

Correlation between miR-200a expression and clinical features of glioma patients. The expression levels of miR-200a in cancer tissue and serum were not correlated with patient age, sex and tumor location $(\mathrm{P}>0.05)$, but were correlated
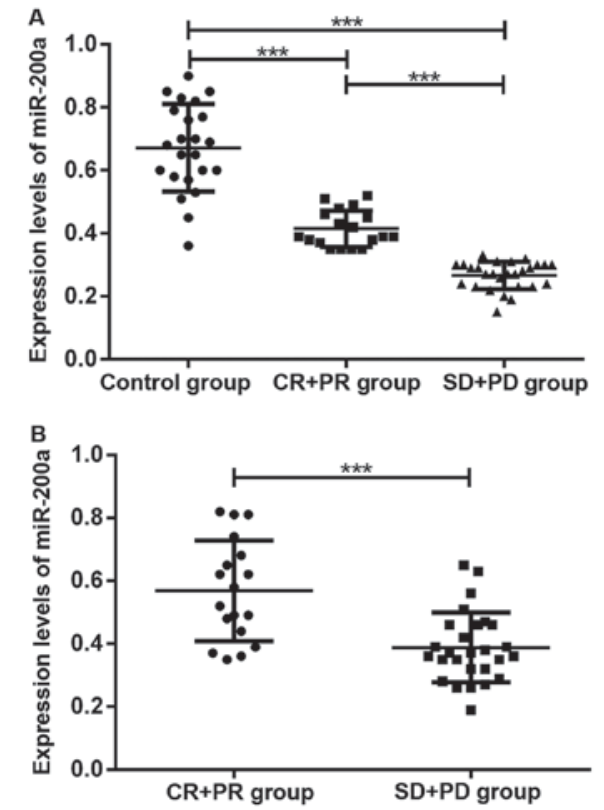

Figure 3. Expression levels of miR-200a before chemotherapy in serum and cancer tissue for different efficacy outcomes after chemotherapy. The expression levels of miR-200a in serum and cancer tissue in chemotherapynon-responsive patients were lower than those in chemotherapy-responsive patients. ${ }^{* * *} \mathrm{P}<0.001$.

with pathological grade and tumor size $(\mathrm{P}<0.05)$. The detailed results were shown in Table II.

Expression levels of miR-200a before chemotherapy in cancer tissue and serum for different efficacy outcomes after chemotherapy. Among 45 patients with high-grade gliomas, 
there were 1, 17, 15 and 12 cases that experienced CR, PR, SD and $\mathrm{PD}$, respectively, after 5 months of chemotherapy treatment. The expression levels of miR-200a in serum and cancer tissue in chemotherapy-non-responsive patients were lower than those in chemotherapy-responsive patients $(\mathrm{P}<0.05)$. The serum levels of miR-200a in chemotherapy-responsive patients were lower than those in healthy subjects in the control group $(\mathrm{P}<0.05)$. As shown in Fig. 3, the expression level of miR-200a was correlated with chemotherapeutic efficacy for treatment of glioma.

\section{Discussion}

With continued advances of human genome studies, it was found that there are only approximately 20,000 gene sequences with coding function, accounting for less than $2 \%$ of the whole genome sequences. Most of the gene sequences are non-coding RNAs, including miRNAs (7). miRNAs are short RNA molecules with 17-25 nucleotides in length and do not have a coding function. miRNAs can inhibit target gene expression by binding to the 3 -untranslated region of the target gene, thereby regulating biological processes including cell proliferation, differentiation, apoptosis and innate immunity (8). A large number of studies suggested that miRNAs may be involved in onset and progression of many cancers, including gliomas, as either oncogenes or tumor suppressor genes (9).

The major members of the miR-200 family that have been discovered so far are miR-200a, miR-200b, miR-200c, miR-141 and miR-429, of which miR-200a, miR-200b and miR-429 are located on chromosome 1, while miR-200c and miR-141 are located on chromosome 12 (10). Among these members, miR-200a played a role as tumor suppressor in a variety of cancers, including gliomas. In nasopharyngeal carcinoma, miR200a expression was downregulated, and the expression level of miR-200a in undifferentiated nasopharyngeal carcinoma cells was significantly lower than that in highly differentiated nasopharyngeal carcinoma cells. As a tumor suppressor, miR200a can inhibit nasopharyngeal carcinoma cell proliferation, invasion and metastasis by attenuating ZEB2 and CTNNB1 expression (11). In liver cancer, miR-200a was downregulated also in cancer tissue. As a tumor suppressor, miR-220a can inhibit cancer cell growth and metastasis by blunting MACC1 gene expression. In addition, the 1-, the 3- and the 5-year survival rates were lower for patients with low miR-200a expression than those with high miR-200a expression (12). In breast cancer, miR-200a was also found to be downregulated in cancer tissue, and as a tumor suppressor, miR-200a can exert an inhibitory effect on growth of breast cancer cells through downregulating TFAM expression (13). miR-200a also played a role in inhibiting gastric adenocarcinoma growth by reducing ZEB1 and ZEB2 expression, inactivating the $\mathrm{wnt} / \beta$-catenin signaling pathway, and attenuating EMT expression (14). For gliomas, Berthois et al (15) reported that the miR-200a expression level in grade IV glioma tissue was lower than those in grade II and III gliomas. Su et al (16) reported that miR-200a was downregulated in glioma tissue, and overexpression of miR-200a in T98G cells reduced glioma cell proliferation, invasion and metastasis. Inhibition of miR-200a expression led to upregulation of SIM-2 expression, thereby promoting tumor growth. Ma et al (17) reported that IncRNA-ATB promoted glioma cell proliferation, invasion and metastasis by inhibiting miR-200a expression. If IncRNA-ATB was inactivated, then miR-200a was re-activated, which lead to reduction of glioma cell proliferation, invasion and metastasis.

Drug resistance of cancer cells in chemotherapy is an important factor attenuating chemotherapeutic efficacy, thereby rendering lower survival rate for cancer patients. Residual cancer cells surviving chemotherapy gradually developed resistance to the therapeutic drug, resulting in failed chemotherapy leading to cancer recurrence (18). Temozolomide is a newly discovered broad spectrum anticancer drug, and it is the safest first-line chemotherapy drug currently used in the treatment of glioma world-wide. Temozolomide is rapidly converted at physiologic $\mathrm{pH}$ to the active form 3-methyl-(triazen-1-yl)imidazole-4-carboxamide (MTIC). Its cytotoxicity is mediated by alkylation of DNA in cancer cells, resulting in apoptosis or necrosis (19). In the mid-to-late phase of chemotherapy for gliomas, varying degrees of drug resistance to temozolomide was developed in some patients, leading to the failure of chemotherapy. Induction of chemotherapy drug resistance is a major factor that compromises chemotherapeutic efficacy (20). With continued advances of gene chip and sequencing technologies, it was found that abnormal miRNA expression was an important factor affecting sensitivity of cancer cells to chemotherapeutic drugs. miRNAs may be involved in onset and progression of cancer cell resistance to chemotherapeutic drugs as either oncogenes or tumor suppressor genes (21). In glioma cell line U87MG, miRNA-21 induced upregulation of Bcl-2 and downregulation of Bax. While in chemotherapy treatment, temozolomide induced cancer cell apoptosis by inhibiting Bcl-2 expression and promoting Bax expression. Therefore, overexpression of miRNA-21 attenuated temozolomide-induced apoptosis of cancer cells, rendering resistance of cancer cells to temozolomide (22). However, overexpression of miR-200a in grade IV glioma cells increased cell sensitivity to temozolomide. In addition, the miR-200a expression level in sensitive glioma cells to temozolomide was significantly higher than that in less sensitive glioma cells to temozolomide (15).

In the present study, the expression levels of miR-200a in cancer tissue, paracancerous tissue and serum of patients with glioma, as well as in serum of healthy subjects were determined using qRT-PCR assay. It was found that the expression level of miR-200a in cancer tissue was significantly lower than that in paracancerous tissue $(\mathrm{P}<0.05)$. In addition, it was also found that the serum level of miR-200a in the observation group was significantly lower than those in the control group $(\mathrm{P}<0.05)$. Correlation between miR-200a expression and clinical features of patients with glioma was explored. It was found that the expression level of miR-200a in serum was correlated with pathological grade and tumor size, but not correlated with the patient's age, sex and tumor location. Thus, it was presumed that miR-200a was involved in onset and progression of glioma. In this study, the efficacy of temozolomide in combination with radiotherapy in treatment of high-grade gliomas was also evaluated. It was found that the expression levels of miR-200a in serum and cancer tissue in chemotherapy-non-responsive patients (SD and $\mathrm{PD}$ ) were lower than those in chemotherapyresponsive patients $(C R$ and $P R, P<0.05)$. The serum level of miR-200a in chemotherapy-responsive patients (CR and PR) 
was lower before chemotherapy than that in healthy subjects in the control group $(\mathrm{P}<0.05)$. Thus, it was presumed that changes in miR-200a expression were correlated with chemotherapeutic efficacy of glioma treatment.

In conclusion, the expression levels of miR-200a in cancer tissue and serum of patients with glioma were reduced. miR-200a expression was correlated with glioma pathological grade, tumor size, as well as chemotherapeutic efficacy, but was not correlated with patient's age, sex and tumor location. Low expression of miR-200a represented involvement in onset and progression of glioma, and was related to cancer cell drug resistance. The results in this study suggest that miR-200a may become a new target for the treatment of gliomas.

\section{Acknowledgements}

Not applicable.

\section{Funding}

No funding was received.

\section{Availability of data and materials}

The datasets used and/or analyzed during the present study are available from the corresponding author on reasonable request.

\section{Authors' contributions}

XW and YL were responsible for the conception and design of the study. CW and LK were responsible for collecting the data. YL and XZ analyzed and interpreted the data. CW drafted this manuscript. XW revised it critically for important intellectual content. All authors read and approved the final manuscript.

\section{Ethics approval and consent to participate}

The study was approved by the Ethics Committee of the Sixth People's Hospital of Jinan (Jinan, China). Signed written informed consents were obtained from the patients and/or guardians.

\section{Consent for publication}

Not applicable.

\section{Competing interests}

The authors declare that they have no competing interests.

\section{References}

1. Bush NA, Chang SM and Berger MS: Current and future strategies for treatment of glioma. Neurosurg Rev 40: 1-14, 2017.

2. Hempel JM, Schittenhelm J, Brendle C, Bender B, Bier G, Skardelly M, Tabatabai G, Castaneda VS, Ernemann U and Klose U: Effect of perfusion on diffusion kurtosis imaging estimates for in vivo assessment of integrated 2016 WHO glioma grades: A cross-sectional observational study. Clin Neuroradiol 1-11, 2017. https://doi.org/10.1007/s00062-017-0606-8.

3. Feng X, Yu Y, He S, Cheng J, Gong Y, Zhang Z, Yang X, Xu B, Liu X, Li CY, et al: Dying glioma cells establish a proangiogenic microenvironment through a caspase 3 dependent mechanism. Cancer Lett 385: 12-20, 2017.
4. Madsen SJ, Christie C, Hong SJ, Trinidad A, Peng Q, Uzal FA and Hirschberg H: Nanoparticle-loaded macrophage-mediated photothermal therapy: Potential for glioma treatment. Lasers Med Sci 30: 1357-1365, 2015.

5. Munoz JL, Rodriguez-Cruz V, Ramkissoon SH, Ligon KL, Greco SJ and Rameshwar P: Temozolomide resistance in glioblastoma occurs by miRNA-9-targeted PTCH1, independent of sonic hedgehog level. Oncotarget 6: 1190-1201, 2015.

6. Su Y, He Q, Deng L, Wang J, Liu Q, Wang D, Huang Q and Li G: MiR-200a impairs glioma cell growth, migration, and invasion by targeting SIM2-s. Neuroreport 25: 12-17, 2014.

7. Ganju A, Khan S, Hafeez BB, Behrman SW, Yallapu MM, Chauhan SC and Jaggi M: miRNA nanotherapeutics for cancer. Drug Discov Today 22: 424-432, 2017.

8. Cheng CJ, Bahal R, Babar IA, Pincus Z, Barrera F, Liu C, Svoronos A, Braddock DT, Glazer PM, Engelman DM, et al: MicroRNA silencing for cancer therapy targeted to the tumour microenvironment. Nature 518: 107-110, 2015.

9. Jonas $\mathrm{S}$ and Izaurralde $\mathrm{E}$ : Towards a molecular understanding of microRNA-mediated gene silencing. Nat Rev Genet 16: 421-433, 2015.

10. Ning X, Shi Z, Liu X, Zhang A, Han L, Jiang K, Kang C and Zhang Q: DNMT1 and EZH2 mediated methylation silences the microRNA-200b/a/429 gene and promotes tumor progression. Cancer Lett 359: 198-205, 2015.

11. Xia H, Ng SS, Jiang S, Cheung WKC, Sze J, Bian XW, Kung HF and Lin MC: miR-200a-mediated downregulation of ZEB2 and CTNNB1 differentially inhibits nasopharyngeal carcinoma cell growth, migration and invasion. Biochem Biophys Res Commun 391: 535-541, 2010

12. Feng J, Wang J, Chen M, Chen G, Wu Z, Ying L, Zhuo Q, Zhang J and Wang W: miR-200a suppresses cell growth and migration by targeting MACC1 and predicts prognosis in hepatocellular carcinoma. Oncol Rep 33: 713-720, 2015.

13. Yao J, Zhou E, Wang Y, Xu F, Zhang D and Zhong D: microRNA200 a inhibits cell proliferation by targeting mitochondrial transcription factor A in breast cancer. DNA Cell Biol 33: 291-300, 2014.

14. Cong N, Du P, Zhang A, Shen F, Su J, Pu P, Wang T, Zjang J, Kang $C$ and Zhang Q: Downregulated microRNA-200a promotes EMT and tumor growth through the wnt/ $\beta$-catenin pathway by targeting the E-cadherin repressors ZEB1/ZEB2 in gastric adenocarcinoma. Oncol Rep 29: 1579-1587, 2013.

15. Berthois Y, Delfino C, Metellus P, Fina F, Nanni-Metellus I, Al Aswy H, Pirisi V, Ouafik L and Boudouresque F: Differential expression of miR200a-3p and miR21 in grade II-III and grade IV gliomas: Evidence that miR200a-3p is regulated by $\mathrm{O}^{6}$-methylguanine methyltransferase and promotes temozolomide responsiveness. Cancer Biol Ther 15: 938-950, 2014.

16. Su Y, He Q, Deng L, Wang J, Liu Q, Wang D, Huang Q and Li G: MiR-200a impairs glioma cell growth, migration, and invasion by targeting SIM2-s. Neuroreport 25: 12, 2014.7.

17. Ma CC, Xiong Z, Zhu GN, Wang C, Zong G, Wang HL, Bian EB and Zhao B: Long non-coding RNA ATB promotes glioma malignancy by negatively regulating miR-200a. J Exp Clin Cancer Res 35: 90, 2016.

18. Li N, Yang L, Wang H, Yi T, Jia X, Chen C and Xu P: MiR-130a and miR-374a function as novel regulators of cisplatin resistance in human ovarian cancer A2780 cells. PLoS One 10: e0128886, 2015.

19. Elhag R, Mazzio EA and Soliman KF: The effect of silibinin in enhancing toxicity of temozolomide and etoposide in p53 and PTEN-mutated resistant glioma cell lines. Anticancer Res 35: 1263-1269, 2015.

20. Prieto-Vila M, Takahashi RU, Usuba W, Kohama I and Ochiya T: Drug resistance driven by cancer stem cells and their niche. Int J Mol Sci 18: E2574, 2017.

21. Wang Q, Wang Z, Chu L, Li X, Kan P, Xin X, Zhu Y and Yang P: The effects and molecular mechanisms of MiR-106a in multidrug resistance reversal in human glioma U87/DDP and U251/G cell lines. PLoS One 10: e0125473, 2015.

22. Shi L, Chen J, Yang J, Pan T, Zhang S and Wang Z: MiR-21 protected human glioblastoma U87MG cells from chemotherapeutic drug temozolomide induced apoptosis by decreasing Bax/ Bcl-2 ratio and caspase-3 activity. Brain Res 1352: 255-264, 2010.

This work is licensed under a Creative Commons Attribution-NonCommercial-NoDerivatives 4.0 International (CC BY-NC-ND 4.0) License. 